\title{
Cell wounding in early experimental acute pancreatitis
}

\author{
Dale E Bockman, Junchao Guo, Michael W Müller, Helmut Friess and Markus W Büchler \\ Department of General Surgery, University of Heidelberg, Germany
}

\begin{abstract}
It is well established that damage to the outer membrane of cells is a common phenomenon allowing abnormal transmission of substances into the cytosol. Penetration of albumin into acinar cells has been detected in experimental acute pancreatitis, raising the possibility that membrane damage is a very early event, potentially representing the first changes leading to pancreatitis. To determine if direct damage to the cell membrane is a key factor during induction of acute pancreatitis, thus altering the balance of extra- and intracellular substances, fluorescein-dextran was administered with supramaximal doses of caerulein via the jugular vein or by injection directly into the pancreas. This tracer rapidly penetrates into cells. Two patterns of tracer penetration are observed: cytosolic and vesicular/vacuolar. Fluorescein-dextran administered intravenously with caerulein penetrates into the cytosol of acinar cells within $10 \mathrm{~min}$. Strong cytoplasmic fluorescence occurs within $5 \mathrm{~min}$ after direct injection. It may be concluded that supramaximal caerulein, administered in vivo, damages the cell membrane of acinar cells, allowing large molecules to enter the cytosol. Thus $\mathrm{Ca}^{2+}$ and other substances may enter the cells in abnormally high concentrations, initiating the cellular changes characteristic of pancreatitis. The results raise the question whether membrane wounding may play a role in the initiation of human pancreatitis.

Laboratory Investigation (2004) 84, 362-367, advance online publication, 9 February 2004; doi:10.1038/labinvest.3700064
\end{abstract}

Keywords: caerulein pancreatitis; membrane wounding; supramaximal caerulein; fluorescein-dextran

Disruption of the plasma membrane challenges the survival of a cell. Severe, unrepaired damage leads to cell death. However, cells have the capacity to restore the integrity of their membrane after less severe damage. They may then resume their normal function.

A variety of mechanical processes cause disruption in the cell membrane. The damage usually is visualized by detecting intracellular localization of tracers that are excluded from the cell if the membrane is intact. ${ }^{1}$ For example, when the plasma membranes of lining cells of the gut are disrupted in vivo, tracers enter the cytosol and are retained there when the membrane reseals, allowing the cells to survive. ${ }^{2}$ Functional studies, using cells from sea urchin eggs to $3 \mathrm{~T} 3$ fibroblasts, have established the capacity to reseal damaged membranes rapidly, and the necessity for $\mathrm{Ca}^{2+}$ to allow this resealing. ${ }^{3,4}$

Wounding of the membrane of some cells, such as muscle cells or lining cells of the gut, might cause

Correspondence: DE Bockman, 3303 Rae's Court, Augusta, GA 30909-3139, USA.

E-mail: hodney@msn.com

Received 28 May 2003; revised 05 August 2003; accepted 27 August 2003; published online 9 February 2004 minor local perturbations. On the other hand, damage to the membrane of acinar cells, with the potential to activate and release potent hydrolytic enzymes, could produce more far-reaching consequences. In order to evaluate the possibility that a very early event in the development of acute pancreatitis involves damage to the membrane of acinar cells, albumin, which normally is excluded from acinar cells, was immunolocalized in rats subjected to experimental acute pancreatitis., ${ }^{5,6}$ Massive cell membrane damage, indicated by cytoplasmic localization of albumin, was detected after retrograde ductal infusion of taurocholate to produce severe acute pancreatitis in rats. Moreover, cytosolic localization of albumin was present after intraperitoneal injection of caerulein to produce mild pancreatitis. Significant cytosolic localization of albumin was detected $15 \mathrm{~min}$ after caerulein injection, although it was not as strong as $30 \mathrm{~min}$ after injection.

An in vitro study of cell damage initiated by supramaximal caerulein led to the conclusion that it is a secondary effect of trypsin activation. ${ }^{7}$ Trypsin was activated within $5 \mathrm{~min}$, but incubation of acini in vitro with caerulein and $\mathrm{Ca}^{2+}$ did not cause membrane damage at $15 \mathrm{~min}$. Cell injury (based on loss of lactic dehydrogenase and incorporation of 
propidium iodide) was first detected $30 \mathrm{~min}$ after exposure. ${ }^{7}$

The evidence of cell damage occurring earlier than $30 \mathrm{~min}$ in vivo led us to explore the possibility that the membrane was wounded by a method other than an attack by trypsin, using well-studied tracers in shorter time periods. The results are consistent with supramaximal caerulein altering the membrane directly, representing an initial effect in experimental acute pancreatitis.

\section{Materials and methods}

Male Sprague-Dawley rats weighing 120-140 g were anesthetized with Ketanest and Rompun. For intravenous administration, the jugular vein was exposed and cannulated. A volume of $1 \mathrm{ml}$ of tracer in phosphate-buffered saline, $\mathrm{pH}$ 7.4, with or without caerulein, was injected, and the pancreas was removed after 5-15 min. For direct injection, the spleen and pancreas were exteriorized through an abdominal incision. Tracer, with or without caerulein, contained in a $100 \mu \mathrm{l}$ microsyringe fitted with a microneedle, was injected into the substance of the pancreas, which was then removed after 5-15 min. Experiments were approved by the animal research committee of the University.

Fluorescein-dextran was obtained from Sigma (DTAF-Dextran, approximately $10000 \mathrm{MW}$ ) and from MoBiTec (Göttingen, Germany; Molecular Probes fluorescein-dextran of approximately 3000, 10000 , or $70000 \mathrm{MW}$ ). The fluorescein-dextran of 3000 and $10000 \mathrm{MW}$ from Molecular Probes was conjugated with biotin to allow localization of the tracer by incubation with avidin-horseradish peroxidase (MoBiTec) followed by diaminobenzidine. Ferritin was purchased from Science Services (Munich, Germany). Trypsin was from Sigma.

For intravenous administration, $50 \mathrm{mg}$ tracer, with or without $20 \mu \mathrm{g}$ ceruletid (Pharmacia \& Upjohn, Erlangen, Germany), was injected during approximately $1 \mathrm{~min}$. Direct injection was with $1 \mathrm{mg}$ tracer with or without $20 \mu \mathrm{g}$ caerulein (Sigma).

This report is based on observations from a total of 63 rats. Fluorescein-dextran was administered intravenously in 27 (16 with caerulein, 11 without). Fluorescein-dextran was administered directly in 17 (nine with caerulein, eight without). Phosphatebuffered saline was injected in six (three intravenously and three directly) to control for fluorescence. To test for the effect of trypsin, nine were injected directly with fluorescein-dextran (five with trypsin, four without). Four were injected directly with ferritin (two with caerulein, two without).

\section{Results}

\section{Penetration of Fluorescein-Dextran}

Fluorescein-dextran of different sizes penetrates into the cytosol of acinar cells when administered with supramaximal doses of caerulein intravenously or directly. The quantity of intracellular tracer varies. Some regions of the pancreas fluoresce brighter than others, presumably due to the regional variability of microcirculation, or to the variable dilution of directly injected tracer. Furthermore, there is variability in the quantity of tracer in cells (Figure 1a). It is common for the cytosolic distribution of the tracer to reveal some organelles, especially nuclei, as negative images (Figure 1a).

Fluorescence is strong 10 (Figure 1a) and $15 \mathrm{~min}$ after intravenous injection of tracer with caerulein. Specimens harvested at similar times after injection of tracer without caerulein reveal fluorescence in vessels and extracellular matrix, with negative acini (Figure 1b).

Similar cytosolic localization of tracer in acinar cells is evident after injection of tracer with caerulein directly into the substance of the pancreas (Figure 1c). Cytosolic localization of fluoresceindextran is accomplished within 5 min of injecting $\sim 70000 \mathrm{MW}$ fluorescein-dextran with caerulein (Figure 1c). In addition to cytosolic distribution, there is clear localization in vesicles, many of which are apical. A few elongate, brightly fluorescent areas represent tracer in lumens.

The dual localization of tracer, cytosolic and vesicular/vacuolar, is more sharply demonstrated by the direct injection of biotinylated fluoresceindextran ( $10000 \mathrm{MW}$ ) for $5 \mathrm{~min}$ (Figure 1d). Localization of the tracer by visualization of the biotin reveals cytosolic localization that varies in intensity from cell to cell, and vesicular localization that appears as dense granules (Figure 1d).

At $10 \mathrm{~min}$ after direct injection with $\sim 3000 \mathrm{MW}$ fluorescein-dextran, the tracer is localized in the cytosol, in vesicles and vacuoles, and in the lumens, without evidence of penetration to the lumen between cells (Figure 1e).

\section{Trypsin does not Mimic Caerulein}

In order to determine if administration of trypsin with tracer led to the same result as administration of caerulein with tracer, $100 \mu \mathrm{l}$ of $0.1 \%$ trypsin was injected directly into the pancreas with fluorescein-dextran. The pancreas was removed $5 \mathrm{~min}$ after injection and processed as for caerulein and tracer. Fluorescence was present in the extracellular matrix and not in the acinar cells (Figure 1f).

\section{Formation of Vacuoles}

The vacuoles that form within minutes after administration of caerulein result from fusion of intracellular vesicles/vacuoles (Figure 2). The peculiar crescent-shaped or ring-shaped fluorescent areas form from the fusion of tracer-containing vesicles/vacuoles to the outside of vacuoles that 
contain no tracer, without immediate mixing. An idea of the fusion sequence may be gained from comparing Figure 2a, an earlier stage, with Figure 2b, in which the fusion process is more advanced. Fusion of vesicles/vacuoles without tracer to a vacuole containing tracer is shown in Figure 2c.

\section{Exclusion of the High-Molecular-Weight Molecule Ferritin}

Direct injection of ferritin, a macromolecule of about $500000 \mathrm{MW}$, with caerulein did not result in its penetration into the cytosol or vacuoles (not shown). It was confined to the extracellular space in a

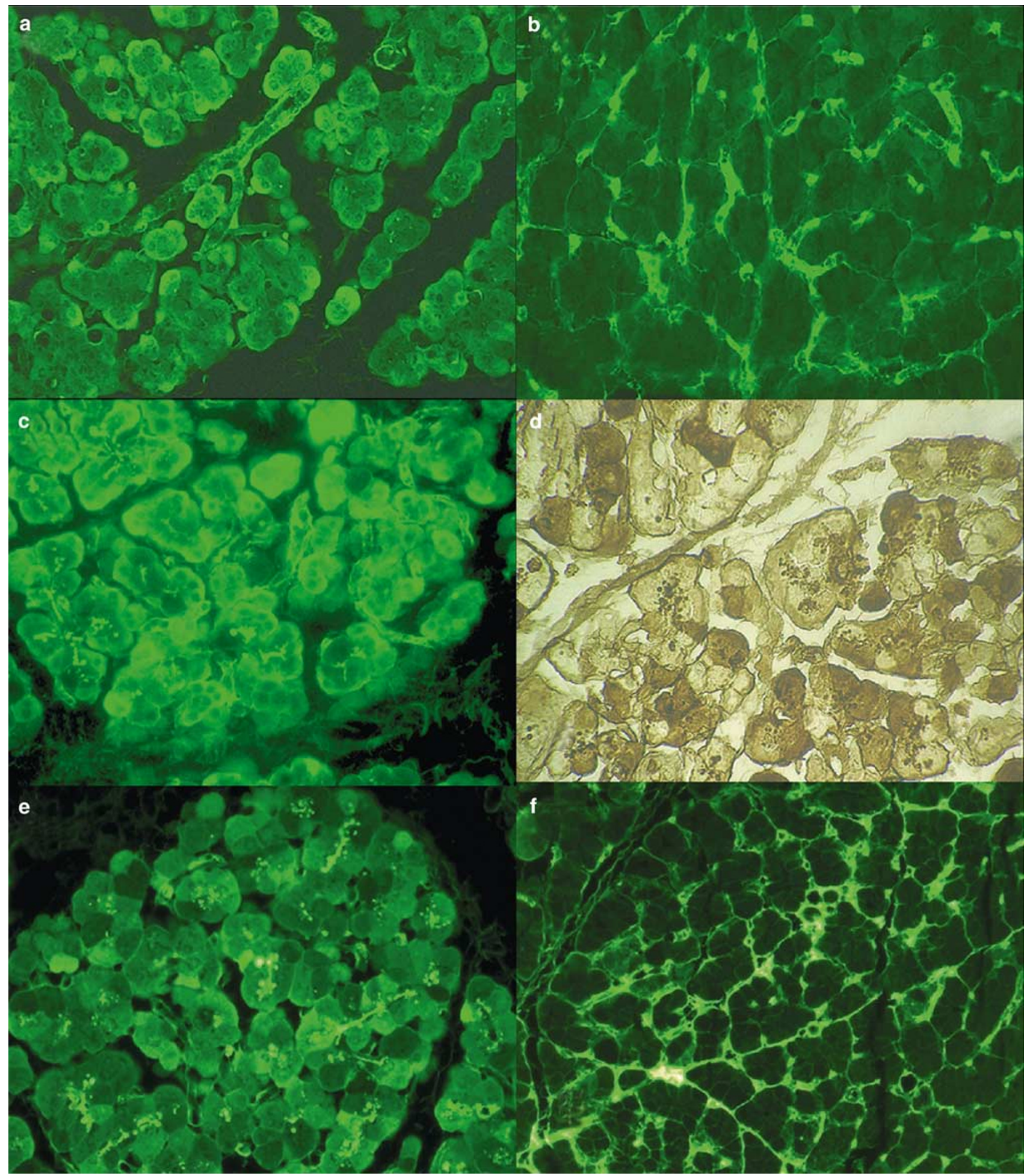




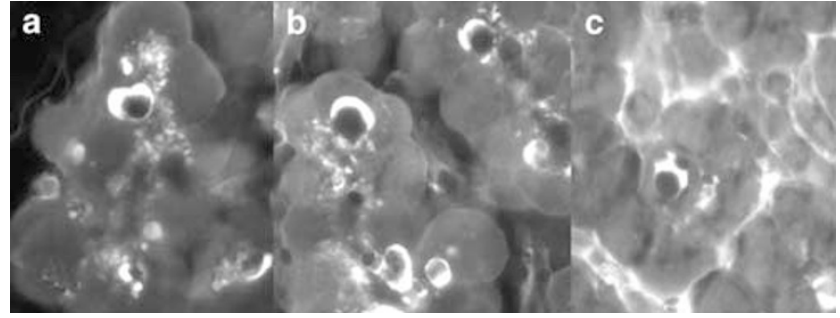

Figure 2 Images of intracellular fusion of vesicles and vacuoles after injection of fluorescein-dextran with caerulein. (a) The disposition of the prominent vacuole suggests the fusion of two tracer-filled vesicles with a vacuole that does not contain tracer. At $10 \mathrm{~min}$ after direct injection of fluorescein-dextran $(\sim 3000 \mathrm{MW})$. Original magnification $\times 1060$. (b) The vacuole to the left of the center is composed of a crescentic area with tracer over an area with no tracer. The vacuole at the top appears to have three tracer-filled vesicles fusing with a tracerless vacuole. Direct injection of fluorescein-dextran $(\sim 3000 \mathrm{MW})$. Original magnification $\times 1060$. (c) The vacuole in the center appears to show the reverse of (a) and (b) in that three tracerless vesicles/vacuoles are fusing with a vacuole containing fluorescein-dextran. At $10 \mathrm{~min}$ after intravenous injection of fluorescein-dextran ( 10000 MW). Original magnification $\times 1060$.

pattern similar to the one in Figure $1 \mathrm{~b}$. The largest molecule to enter the cytosol in the current study was the $\sim 70000 \mathrm{MW}$ fluorescein-dextran.

\section{Discussion}

This study reveals the incorporation of membraneimpermeant molecules into the cytosol of acinar cells during the very early stages of experimental acute pancreatitis induced by administration of supramaximal caerulein. Furthermore, it establishes that material from the extracellular medium (revealed by using tracers) may also be incorporated into intracellular vesicles and vacuoles, and that this material can be transported to the acinar lumen by transcytosis. It is proposed that an altered plasma membrane allows the massive influx of $\mathrm{Ca}^{2+}$ from the extracellular medium that has been shown to accompany supramaximal stimulation, thus comprising a central initiating event. The study demonstrates directly and convincingly the membrane changes within the cell that are a part of the process by which the cells are altered in the earliest stages of experimental acute pancreatitis, presumably due to alteration of the plasma membrane. The significant observation is that, contrary to the belief that only intracellular vesicles and vacuoles fuse in response to caerulein stimulation (and therefore only substances previously contained within a normal cell intermix), extracellular substances taken into the cell rapidly intermix with intracellular substances. The addition of extracellular substances to the vacuoles increases significantly the range of effects possible.

Acinar cells secrete by $\mathrm{Ca}^{2+}$ - regulated exocytosis. Physiological levels of cholecystokinin or caerulein induce oscillating calcium currents causing zymogen granules to fuse with the apical membrane and release their contents into the acinar lumen. Supramaximal levels, however, produce an influx of extracellular calcium ions leading to higher and sustained intracellular calcium ion concentration $\left(\left[\mathrm{Ca}^{2+}\right]_{\mathrm{i}}\right)$, and fusion of zymogen granules to produce vacuoles. ${ }^{8,9}$ The current demonstration that supramaximal caerulein alters the cell membrane sufficiently to allow molecules up to $\sim 70000 \mathrm{MW}$ to enter the cytosol implies that calcium ions, driven by the tremendous excess extracellular concentration compared with the normal intracellular concentration, can easily enter the cell. Under these conditions, it is not necessary for $\mathrm{Ca}^{2+}$ to enter through calcium channels.

Acinar cells are not unique in displaying membrane wounding, accompanied by penetration of tracers into the cytosol and $\mathrm{Ca}^{2+}$-regulated events, including intracellular membrane fusion. It is not uncommon for the membrane of cells to be disrupted. Furthermore, it is not uncommon for these cells to survive, because they are capable of resealing the injury using fused membranes. Plasma membrane disruptions produced by a variety of means, both natural and experimental, are followed rapidly by $\mathrm{Ca}^{2+}$ influx from the extracellular compartment, vesicle fusion, and membrane repair. ${ }^{10}$

Aggregated lysosomes of cultured mammalian cells are induced to fuse with one another by elevated internal $\mathrm{Ca}^{2+}$ levels. ${ }^{11}$ Permeabilization of the cell membrane with streptolysin-O, allowing extracellular $\mathrm{Ca}^{2+}$ to rush in until the $\left[\mathrm{Ca}^{2+}\right]_{\mathrm{i}}$ is $\sim 1 \mu \mathrm{M}$, causes fusion of lysosomes. Lysosomes also fuse exocytotically with the plasma membrane. ${ }^{12}$ Lysosome membrane marker appears in the patch after resealing of the original membrane defect. ${ }^{13}$

Figure 1 Histological sections of the pancreas using tracers to detect cell wounding and transcytosis. (a) Fluorescence in the cytosol of acinar cells from an animal killed $10 \mathrm{~min}$ after intravenous injection of fluorescein-dextran $(\sim 10000 \mathrm{MW})$ with caerulein, indicating damage to the plasma membrane. Original magnification $\times 180$. (b) Fluorescence in the pancreas of an animal killed 10 min after intravenous injection of fluorescein-dextran $(\sim 10000 \mathrm{MW})$, but with phosphate-buffered saline instead of caerulein. The fluorescence is extracellular, essentially limited to blood vessels and extracellular matrix. Original magnification $\times 360$. (c) At 5 min after injection of fluorescein-dextran ( $\sim 70000 \mathrm{MW})$ with caerulein directly into the pancreas, fluorescence is in the cytosol as well as in vesicles, some of which are in the apex of acinar cells. Original magnification $\times 360$. (d) Pancreas 5 min after direct injection of biotinylated fluoresceindextran ( $10000 \mathrm{MW})$, allowing localization of the tracer using avidin-horseradish peroxidase. Reaction product is in the cytosol of some cells. In addition, vesicles with dense reaction product are present, mirroring the dual type of localization seen in (c). Original magnification $\times 360$. (e) At 10 min after direct injection of fluorescein-dextran $(\sim 3000 \mathrm{MW})$, it is located in the cytosol as well as in vesicles and vacuoles of acinar cells. Some tracer is in the lumen, but intercellular localization is not evident, indicating transcytosis rather than intercellular penetration. Original magnification $\times 180$. (f) At $5 \mathrm{~min}$ after direct injection of fluorescein-dextran $(\sim 70000 \mathrm{MW})$ with trypsin, the tracer is limited to an extracellular distribution, indicating that extracellular trypsin does not cause the early membrane damage. Original magnification $\times 180$. 
Following treatment by supramaximal caerulein, tracers may enter by endocytosis to be contained within vesicles. They may also enter through membrane disruptions, to be secondarily sequestered in vacuoles by membrane fusion as has been demonstrated in other systems. ${ }^{14}$

Penetration of tracer into acinar cells is demonstrated in the current study after administration of tracer with caerulein intravenously and by injection directly into the pancreatic extracellular matrix. Direct injection into the pancreas provides a system that, like in vitro preparations of acini, allows immediate contact of agents with cell membranes, but differs in that the natural environment is maintained, including a blood supply, nerves, and molecules not present in incubation media. The mincing, treatment with collagenase, filtering, and centrifugation necessary to prepare acini for in vitro studies are traumatic. If the acini are kept for extended periods after preparation, there is no certainty that the membranes remain the same as in vivo, but it is certain that the extracellular environment is not exactly the same as in vivo. There is membrane turnover as a result of the trauma, and surface receptors might be lost. There is ample evidence in the literature that mechanical trauma damages the plasma membrane and that repair is accomplished in part by contributions from intracellular membranes. Administering caerulein and tracer intravascularly and into the tissue directly is a gentler method that is closer to normal conditions, and the time periods are much shorter than can be accomplished with preparation of acini to be studied in vitro. Direct injection circumvents the necessity for transmission of caerulein and tracer through vascular walls, with whatever delay and screening that might entail.

Trypsin is activated in acini by supramaximal caerulein and carbamylcholine. $\mathrm{Ca}^{2+}$ is necessary for this to occur. ${ }^{7}$ A sustained rise in $\left[\mathrm{Ca}^{2+}\right]_{\mathrm{i}}$ induces trypsin activation that at $15 \mathrm{~min}$ after the start of hyperstimulation with cholecystokinin, is essentially limited to the apical pole. Zymogen granules are largely replaced by vacuoles. ${ }^{9}$ The results of the current study show that injection of trypsin with tracer does not reproduce the penetration of the tracer that is observed when it is injected with caerulein, indicating that the alteration by caerulein is likely not dependent secondarily on trypsin activation. The results of the current study provide an expanded explanation for cellular events that occur during development of mild experimental pancreatitis. The membrane damage ascribed by others to the secondary effects of intracellularly activated trypsin occurs later than the membrane wounding observed in the current study. This is interpreted as evidence that membrane wounding occurs prior to trypsin activation. This interpretation provides a mechanism by which intracellular events, including enzyme activation, are initiated.
Administration of supramaximal caerulein causes '...severe changes in the specificity of the intracellular membrane fusion process....' leading to fusion of zymogen granules. ${ }^{8}$ The results of the current study clearly indicate that alteration of the plasma membrane of acinar cells allows substances at least as large as $70000 \mathrm{MW}$ to enter the cytosol. It is proposed that a significant event as a result of membrane alteration is the influx of $\mathrm{Ca}^{2+}$, leading to fusion of zymogen granules and lysosomes. This is entirely consistent with investigations of cell wounding and of acinar cell response to caerulein. The mechanism by which caerulein alters the membrane sufficiently to admit external molecules remains to be determined. The sustained cell injury seen after supramaximal caerulein would seem to include, but not be limited to, trypsinogen activation.

These results predict that agents that alter the plasma membrane of acinar cells sufficiently to allow the penetration of molecules that normally would be excluded would have the same effect as supramaximal caerulein. They raise the question as to whether excessive neural stimulation and carbamylcholine administration would produce similar results.

\section{Acknowledgement}

The first two authors contributed equally to this manuscript. DEB, Professor and Chairman Emeritus, Department of Cellular Biology and Anatomy, Medical College of Georgia, Augusta, was Visiting Professor in the Department of General Surgery, University of Heidelberg. JG was Research Fellow from the Department of General Surgery, Peking Union Medical College Hospital, Chinese Academy of Medical Science \& Peking Union Medical College, Beijing, PR China. This work was supported by a Mercator Guest Professorship from the Deutsche Forschungsgemeinschaft (for DEB and JG).

\section{References}

1 McNeil PL, Steinhardt RA. Loss, restoration, and maintenance of plasma membrane integrity. J Cell Biol 1997;137:1-4.

2 McNeil PL, Ito S. Gastrointestinal cell plasma membrane wounding and resealing in vivo. Gastroenterology 1989;96:1238-1248.

3 McNeil PL, Baker MM. Cell surface events during resealing visualized by scanning-electron microscopy. Cell Tiss Res 2001;304:141-146.

4 Steinhardt RA, Bi G, Alderton JM. Cell membrane resealing by a vesicular mechanism similar to neurotransmitter release. Science 1994;263:390-393.

5 Müller MW, McNeil PL, Büchler MW, et al. Membrane wounding of acinar cells is an early event in experimental acute pancreatitis. Pancreas 1995;11:442 (Abstract). 
6 Müller MW, McNeil PL, Büchler MW, et al. Membrane wounding and early ultrastructural findings. In: Büchler MW, Uhl W, Friess H, Malfertheiner P (eds.) Acute Pancreatitis: Novel Concepts in Biology and Therapy. Blackwell: Berlin, 1999, pp. 27-34.

7 Saluja AK, Bhagat L, Lee HS, et al. Secretagogueinduced digestive enzyme activation and cell injury in rat pancreatic acini. Am J Physiol 1999;276: G835-G842.

8 Adler G, Rohr G, Kern HF. Alteration of membrane fusion as a cause of acute pancreatitis in the rat. Dig Dis Sci 1982;27:993-1002.

9 Raraty M, Ward J, Erdemli G, et al. Calcium-dependent enzyme activation and vacuole formation in the apical granular region of pancreatic acinar cells. Proc Natl Acad Sci 2000;97:13126-13131.
10 McNeil PL. Repairing a torn cell surface: make way, lysosomes to the rescue. J Cell Sci 2002;115:873-879.

11 Bakker AC, Webster P, Jacob WA, et al. Homotypic fusion between aggregated lysosomes triggered by elevated $\left[\mathrm{Ca}^{2+}\right]_{i}$ in fibroblasts. J Cell Sci 1997;110: 2227-2238.

12 Rodriguez A, Webster P, Ortego J, et al. Lysosomes behave as $\mathrm{Ca}^{2+}$-regulated exocytic vesicles in fibroblasts and epithelial cells. J Cell Biol 1997;137:93-104.

13 Reddy A, Caler EV, Andrews NW. Plasma membrane repair is mediated by $\mathrm{Ca}^{2+}$-regulated exocytosis of lysosomes. Cell 2001;106:157-169.

14 Terasaki M, Miyake K, McNeil PL. Large plasma membrane disruptions are rapidly resealed by $\mathrm{Ca}^{2+}$ dependent vesicle-vesicle fusion events. J Cell Biol 1997;139:63-74. 